\title{
Estudo sobre a comparação das perceções das distâncias espaciais: Realidade virtual versus ambiente real
}

\section{Study on the comparison of perceptions of spatial distances: Virtual reality versus natural environment}

ARTIGO ORIGINAL | ORIGINAL ARTICLE

\begin{abstract}
RESUMO
O objectivo do presente foi comparar diferentes contextos de avaliação de distâncias (sala virtual vs sala real) ao nível da percepção de distâncias. Foi, ainda, avaliado o feito da presença (ou não) de uma mão virtual no cenário de realidade virtual no sentimento de presença. Foram estudados 36 indivíduos (11 mulheres e 25 homens). Os resultados evidenciam que não há diferenças estatisticamente significativas na percepção das distâncias. A presença de uma mão virtual influencia negativamente o sentimento de presença nos contextos virtuais. Concluímos que o uso da realidade virtual é um meio adequado para treinar a perepção/ avaliação de distâncias.
\end{abstract}

Palavras-chave: perceção das distâncias, realidade virtual, ambiente real, membros virtuais

\begin{abstract}
The purpose of the present study was to compare different contexts of distance assessment (virtual room vs. real room) at the level of perception of distances. It was also evaluated the fact of the presence (or not) of a virtual hand in the scenario of virtual reality in the feeling of presence. Thirty-six individuals ( 11 women and 25 men) were studied. The results show that there are no statistically significant differences in the perception of distances. The presence of a virtual hand negatively influences the feeling of presence in virtual contexts. We conclude that the use of virtual reality is an adequate means to train the perception / assessment of distances.
\end{abstract}

Keywords: distance perception, virtual reality, real environment, virtual members 
$\mathrm{Na}$ área da perceção visual, a tecnologia de realidade virtual facilita a criação de eventos de caracter imersivo para estudar a perceção espacial. O estudo sobre perceção das distâncias em ambientes virtuais tem-se concentrado na determinação de quão bem a perceção da distância nesses ambientes corresponde à do ambiente real (Blascovich \& Loomis, 1999; Ziemer, Plumert, Cremer, \& Kearney, 2009).

Ao longo dos últimos quinze anos, os investigadores têm usado sistemas de head mounted-display (HDM's) em ambientes virtuais, para o estudo da perceção do espaço, pois permitem que os participantes se movimentem dentro do ambiente virtual e os estudos têm evidenciado uma tendência para a subestimação da distância em ambientes virtuais em relação a avaliações feitas em contexto real (Creem-Regehr, Stefanucci, Thompson, Nash, \& MacCardell, 2015; Ziemer et al., 2009).

A pesquisa sugere que há diferenças na perceção humana entre ambientes reais e virtuais. Alguns estudos sobre avaliações da distância egocêntrica relatam que os observadores subestimaram essas distâncias em comparação com as suas contrapartes físicas (Creem-Regehr, Gooch, Thompson \& Willemsen, 2008; Matsushima, Vaz, Cazuza, \& Filho, 2014). Uma subestimação da distância num ambiente virtual provavelmente distorce a representação espacial em larga escala, o que, por sua vez, pode limitar o grau em que a informação espacial, em larga escala, obtida num ambiente virtual se transfere para o mundo real. Uma explicação comum para a subestimação é o campo de visão relativamente pequeno na maioria dos sistemas HDM's, mas estudos recentes sugerem que este não é o caso desde que os participantes sejam capazes de olhar ao redor do ambiente (Darken, Krebs, McCarley, Rowland \& Sinai, 1999; Creem-Regehr et al., 2008). Vários estudos concluíram que a perceção de distância é completamente exata dentro do espaço de ação num contexto real (Ziemer et al., 2009). Creem-Regehr et al.
(2015) encontraram diferenças nas avaliações de distância entre os ambientes internos e externos, existindo uma maior subestimação em ambientes externos.

É possível observar que a perceção espacial é suscetível às influências dos estados psicológico e fisiológico do observador, sendo que vários estudos descrevem influências sociais, cognitivas ou ativas (Takahashi, Meilinger, Watanabe, \& Bülthoff, 2013; Jung, et al., 2016). Embora os fatores que influenciam a precisão das estimativas de distância em ambientes virtuais ainda não tenham sido nitidamente reconhecidos (Interrante, Ries, Lindquist, \& Anderson, 2008), alguns fatores que poderão influenciar a precisão das estimativas de distâncias na realidade virtual são inumerados por vários autores. Por exemplo, o estudo de Ziemer et al. (2009) mostrou que a ordem em que as pessoas fazem avaliações de distâncias em ambientes reais e virtuais afeta as suas estimativas, sendo que quando os participantes são expostos em primeiro lugar ao ambiente real as suas estimativas tornam-se mais precisas. Interrante et al. (2008) descobriram que as pessoas realizam avaliações mais precisas sobre as distâncias egocêntricas em ambientes virtuais quando estes apresentam um modelo de alta-fidelidade do espaço físico em que o participante se encontra, sendo que uma interpretação deste facto seria o de que os participantes apresentam maior capacidade de avaliação de distância egocêntrica em ambiente virtual se aceitam o mundo virtual como equivalente ao real. Porém existe uma outra interpretação que argumenta que os participantes são capazes de fazer avaliações precisas de distâncias egocêntricas no ambiente virtual porque foram capazes de formar um modelo mental preciso da estrutura espacial do ambiente real após uma breve exposição a este.

Quando se fala em realidade virtual, existe um conceito central: a presença. Esta refere-se ao sentimento físico de estar no ambiente virtual, percebendo-o como real. O self-avatar 
apresenta o benefício de fornecimento de pistas ao utilizador sobre a localização do corpo no mundo virtual e a sua postura corporal atual. A presença do avatar tem mostrado trazer evidências positivas para as avaliações. Fornecer um avatar, supostamente, corrige estimativas de distância uando o utilizador interpreta o avatar como sendo o seu próprio corpo. Neste sentido, vários autores argumentam que um maior senso de presença pode melhorar a perceção de distância (Renner, Velichkovsky, \& Helmert, 2013; Steed, Pan, Zisch, \& Steptoe, 2016; Westhoven, Paul, \& Alexander, 2016). Por exemplo, os resultados do estudo de Mohler e Bülthoff (2008) fornecem evidência que se os participantes forem capazes de explorar um avatar, totalmente articulado de si, cometerão menos erros nas avaliações subsequentes de distância egocêntrica. Porém a questão da influência do self-avatar sobre a perceção das distâncias é complexa e as opiniões divergem entre os vários autores (Renner, et al., 2013).

É possível que um número de fatores como a resolução da tela, o campo de visão, e a fidelidade de "renderização", podem produzir erros observados na perceção de distância. Se a qualidade dos gráficos for insuficiente pode gerar uma subestimação da distância, mas esta hipótese permanece sem resposta. Possivelmente, por causa da alta qualidade dos gráficos de computadores modernos, muitas vezes é assumido que os seres humanos percebem e comportam-se de forma semelhante em ambientes reais e virtuais. Outro fator que poderá influenciar a perceção da distância, mas para o qual não existem evidências, é o feedback dentro de um ambiente virtual imersivo que pode causar uma distância visualmente direcionada (Creem-Regehr, Mohler \& Thompson, 2006; Knapp \& Loomis, 2004; Renner, et al., 2013; Riecke, Behbahani, \& Shaw, 2009). Relativamente ao sexo e à idade, estas não parecem influenciar as estimativas de distância em ambientes virtuais. Já o nível de experiência com a realidade virtual, em geral, pode ser uma influência na perceção de distâncias (Renner, et al., 2013).

A perceção da distância não pode ser medida diretamente. Em vez disso, avaliações verbais de distância são comumente usadas para fazer inferências sobre a perceção de distância. As distâncias egocêntricas são normalmente subestimadas por medidas verbais explícitas (Hammel, Kelly, Siegel, \& Sjouland, 2014; Li, Philips, \& Durgin, 2011). A estimativa verbal é assumida por alguns autores como um relato preciso de uma representação percetual, enquanto outros os consideram sujeitos a uma variedade de influências cognitivas (Andre $\&$ Rogers, 2006). Através de óculos de realidade virtual, acede-se a essa realidade virtual e o rastreamento da cabeça é usado para atualizar o campo de visão virtual de acordo com o movimento que o utilizador faz com a cabeça (Westhoven, et al., 2016).

O estudo de Alexandrova, Teneva, Rosa, Kloos, Bülthoff e Mohler (2010) consistiu em ver se as distâncias também eram subestimadas num ambiente virtual imersivo numa tela grande (LSDI). Neste estudo, os autores tiveram em consideração as estimativas verbais como medida de resposta. Os resultados demonstraram que as estimativas das distâncias no mundo real eram verídicas, enquanto que no ambiente virtual, as distâncias eram significativamente subestimadas.

Desta forma, o presente estudo centra-se na questão: será que existe maior subestimação das distâncias espaciais na condição "sala real" ou na condição "sala virtual"? Sendo que pretendemos comparar as condições "sala real" e "sala virtual" em função da assertividade das estimativas das distâncias espaciais, de forma a verificar em qual das condições as estimativas são feitas com maior grau de certeza. Assim como, comparar as subcondições da condição "sala virtual", isto é, a subcondição "sala virtual com membros virtuais (com mãos)" e "sala virtual sem membros virtuais (sem mãos)" em função da assertividade das estimativas 
das distâncias espaciais, de forma a verificar em qual das subcondições as estimativas são feitas com maior rau de certeza, na medida em que a literatura menciona que quem visualiza membros virtuais apresenta um maior sentido de presença e por conseguinte avaliará com maior acuidade as distâncias.

Face ao exposto, no presente estudo, as variáveis independentes são a sala virtual, sala real e a presença de mão virtual. Já dependentes foram sentido de presença e as estimativas verbais das distâncias. Desta forma, pretendemos omparar os efeitos que a sala virtual e sala real apresentam nas estimativas das distâncias dos participantes, assim como, os efeitos que os membros virtuais apresentam nas estimativas de distância, isto é, se o sentido de presença na realidade virtual influencia as estimativas de distância, sendo que para isso o sentido de presença será medido em função da presença espacial, envolvimento, realismo experienciado e presença global.

\section{MÉTODO}

O estudo realizado apresenta-se como transversal, é do tipo quase-experimental, devido a não cumprir o requisito de a amostra ser aleatória, sendo que a nossa amostra foi obtida por conveniência. Desenvolvemos, assim, um estudo quantitativo com base em comparações.

\section{Amostra}

Participaram um total de 36 indivíduos (11 do sexo feminino e 25 do sexo masculino), todos residentes no interior da Região Norte de Portugal, com idade média de 21.47 (DP = .375), onde o mínimo foi 18 e o máximo foi 27. Todos frequentam o ensino superior, e $69.4 \%$ usa óculos ou lentes de contato. Relativamente a ter experiência com computadores, $72.2 \%$ apresenta uma experiência básica e $27.8 \%$ apresenta uma boa experiência. Quanto ao conhecimento com a realidade virtual $8.3 \%$ dos participantes não apresenta conhecimento, $77.8 \%$ apresenta um conhecimento básico e $13.9 \%$ um bom conhecimento, sendo que $52.8 \%$ dos participantes nunca teve qualquer experiência ou contacto com óculos de realidade virtual.

\section{Instrumentos}

Os instrumentos usados para a realização do estudo foram uma sala real com $7 \times 7$ metros com uma área útil de aproximadamente $3 \times 3$ metros, 3 cubos definidos como objetos para a perceção de distâncias, e um computador com as propriedades RAM 32GB, processador Intel i7-5820k, 3x NVIDIA GTX 980, e armazenamento de 256 GB. A partir do programa Unity foi criada a sala em modo virtual, que permitiu aos participantes visualizarem os membros virtuais, assim como os cubos. Como HMD utilizou-se os Oculus Rift DK 2 e para a visualização dos membros virtuais foi utilizado um sistema de tracking, nomeadamente o Optitrack que foi adquirido através da colocação de marcadores numas luvas que foram devidamente rastreadas pelo sistema de forma a conseguirmos replicar as mãos e os seus movimentos no ambiente virtual. Usou-se, também, um questionário sociodemográfico, onde uma parte deste era preenchido pelos investigadores que registavam as estimativas das distâncias declaradas pelos participantes. No final da experiência foi preenchido um questionário no final da experiência da condição "sala virtual", de forma a medir o sentido de presença dos participantes com e sem membros virtuais, sendo este o "iGroup Presence Questionnaire" (IPQ), adaptado para a Versão Portuguesa por Vasconcelos-Raposo, t al., \& Sousa (2016). Por fim, utilizou-se o programa IBM SPSS Statistics Versão 23 para analisar os resultados.

\section{Procedimentos}

O nosso estudo foi realizado num laboratório MASSIVE no norte de Portugal. A estrutura laboratorial apresentou duas condições: condição "sala real" e condição "sala virtual". $\mathrm{Na}$ condição "sala real”, participaram 12 estudantes universitários. A condição "sala virtual" 
subdividiu-se em duas condições: "sala virtual com membros virtuais" e "sala virtual sem membros virtuais". Em cada uma destas subcondições participaram 12 estudantes universitários.

A recolha de dados foi feita em duas sessões, respetivamente em dois dias diferentes, na duração de duas horas cada, quatro horas totais. Inicialmente, os participantes leram e preencheram um consentimento informado, sendo que participaram na experiência sem qualquer recompensa. Posteriormente preencheram um questionário sociodemográfico onde estão presentes questões sobre a idade, profissão, género, habilitações académicas e sobre a sua experiência com computadores e com a realidade virtual. Todos os participantes tinham que fazer uma estimativa verbal da distância a que os objetos estariam deles próprios, onde essas distâncias, na realidade foram colocadas a $1.80,2.20$, e 4.80 metros do local de observação. Na condição "sala real", os participantes viram os objetos e fizeram a sua estimativa, e na condição "sala virtual" os participantes colocaram os óculos de realidade virtual, observando a réplica da sala real, e de seguida fizeram as suas estimativas de distância. Em ambas as condições referidas acima as estimativas foram anotadas pelos investigadores. A diferença entre as subcondições da "sala virtual" foi que numa das condições os participantes fizeram as estimativas, com o recurso a visualização de membros virtuais, enquanto na outra subcondição os participantes não visualizaram os membros virtuais. Na condição "sala virtual", no final da experiência, os participantes preencheram um questionário após experiência sobre o sentimento de presença (IPQ), que é composto por 14 questões respondidas em função de uma escala de Likert de 5 pontos que vai de "discordo totalmente" a "concordo totalmente" e aborda as variáveis presença espacial (sentimento de estar fisicamente presente no ambiente virtual), envolvimento (atenção prestada ao ambiente virtual e envolvimento experienciado), realismo experienciado (experiência subjetiva de realismo experienciado no ambiente virtual) e presença global (sensação global de estar presente no ambiente virtual). Os participantes tiveram liberdade para mover a cabeça, de maneira a observar da melhor forma a sala em que se encontravam, sendo que só se aplicou a condição de não mover o corpo do sítio.

\section{Análise Estatística}

Inicialmente, usamos o teste Levene recorrendo ao programa IBM SPSS Statistics Versão 23 para verificar a homogeneidade das variâncias. Utilizando o mesmo programa, recorremos a uma Anova de forma a comparar as distâncias entre a condição real e a condição virtual. A partir da análise de variância unidirecional, comparámos as estimativas, de modo a verificar onde havia maior subestimação. Também a partir da análise de variância unidirecional, chegou-se aos resultados sobre a influência da condição "sala virtual com mãos" versus "sala virtual sem mãos" no sentimento de presença.

\section{RESULTADOS}

A partir da estatística de Levene, a homogeneidade dos dados relativos às três distâncias foi verificada (primeira distância com uma estatística de teste igual a .571 e um valor de $p$ $=.570$; segunda distância com uma estatística de teste igual a 1.130 e um valor de $p=.335$; terceira distância com uma estatística de teste igual a 1.588 e um valor de $p=.220$ ).

A distância a que o primeiro objeto se encontrava do participante foi de 1.80 metros e as estimativas verbais sobre a mesma revelaram uma média de 1.47 metros (desvio padrão = .416) na condição "sala real", uma média de $1.38 \pm .433 \mathrm{~m}$, na condição "sala virtual sem membros virtuais" e uma média de $1.52 \pm$ $.591 \mathrm{~m}$, na condição "sala virtual com membros virtuais" (ver quadro 1).

A distância ao segundo objeto foi 2.20 metros e as estimativas verbais revelaram 
Quadro 1

Médias das estimativas das distâncias para cada objeto em cada condição

\begin{tabular}{lcccc}
\hline & Objeto 1 & Objeto 2 & Objeto 3 & N \\
\hline Condição 1 & 1.47 & 2.57 & 3.45 & 12 \\
Condição 2 & 1.38 & 2.79 & 4.79 & 12 \\
Condição 3 & 1.52 & 2.66 & 4.07 & 12 \\
\hline
\end{tabular}

Nota: A condição 1 refere-se a condição "sala real". A condição 2 refere-se a condição "sala virtual sem membros virtuais" e a condição 3 refere-se a "sala virtual com membros virtuais".

na condição "sala real" uma média de $2.57 \pm$ $.500 \mathrm{~m}$, na condição "sala virtual sem membros virtuais" uma média de $2.79 \pm .689 \mathrm{~m}$ e na condição "sala virtual com membros virtuais" uma média de $2.66 \pm 0.669$ ) (ver quadro 1 ).

A distância ao terceiro objeto foi de 4.80 metros e as estimativas verbais sobre esta primeira distância revelaram uma média de $3.45 \pm 0.837 \mathrm{~m}$, na condição "sala real”, uma média de $4.79 \pm 2.435 \mathrm{~m}$, na condição "sala virtual sem membros virtuais" e uma média de $3.97 \pm 1.081 \mathrm{~m}$ e na condição "sala virtual com membros virtuais" (ver quadro 1).

A partir da ANOVA, foi possível observar que não houve diferenças estatisticamente significativas nas estimativas das distâncias no ambiente real e virtual, sendo que se obteve para a primeira distância entre grupos (combinado) um valor de $p$ $=0.771$ e um valor de $F=0.262$, para a segunda distância entre grupos (combinado) um valor de $p$ $=0.678$ e um valor de $F=0.393$, e para a terceira distância entre grupos (combinado) um valor de $p$ $=0.137$ e um valor de $F=2.114$.

O teste de igualdade de variância do erro de Levene mostra que a variância do erro da variável dependente (estimativas das estimativas verbais) é igual entre os grupos.
Relativamente às estimativas das distâncias e ao sentimento de presença, a presença espacial revelou uma média igual a $2.431 \pm$ $0.321 \mathrm{~m}$ na condição "sala virtual com membros virtuais" e uma média a $2.181 \pm 0.441 \mathrm{~m}$ na condição "sala virtual sem membros virtuais". Para o envolvimento, na condição "sala virtual sem membros virtuais" obteve-se uma média a $2.354 \pm 3.154 \mathrm{~m}$ e na condição "sala virtual com membros virtuais" obteve-se uma média a $0.729 \pm 3.174 \mathrm{~m}$. O realismo revelou uma média igual a $3.083 \pm 0.615 \mathrm{~m}$ na condição "sala virtual com membros virtuais" e uma média a $2.896 \pm$ $0.494 \mathrm{~m}$ na condição "sala virtual sem membros virtuais". A presença global revelou uma média igual a $2.081 \pm 1.178 \mathrm{~m}$, na condição "sala virtual com membros virtuais" e uma média a $2.477 \pm 1.171 \mathrm{~m}$ na condição "sala virtual sem membros virtuais" (ver quadro 2).

A presença espacial apresentou uma média global de $2.306 \pm 0.398 \mathrm{~m}$, o envolvimento uma média global de $1.542 \pm 3.204 \mathrm{~m}$, e o realismo uma média global de $2.989 \pm .554 \mathrm{~m}$. A presença global apresentou uma média global de $2.279 \pm 1.166 \mathrm{~m}$.

A partir do teste de efeitos entre sujeitos, observou-se que não houve efeitos estatís-

Quadro 2

Médias das variáveis do sentido de presença em cada condição

\begin{tabular}{lcccc}
\hline & Realismo & Envolvimento & Presença Espacial & Presença Global \\
\hline Condição 1 & 2.896 & 2.354 & 2.181 & 2.477 \\
Condição 2 & 3.083 & 0.729 & 2.431 & 2.081 \\
\hline
\end{tabular}

Nota: A condição 1 refere-se a condição "sala real". A condição 2 refere-se a condição "sala virtual sem membros virtuais" e a condição 3 refere-se a "sala virtual com membros virtuais". 
ticos significativos, sendo que, com origem na condição, a presença espacial apresentou um $F$ $=2.52$ e um $p=0.13$, o envolvimento um $F=$
1.58 e um $p=0.22$, o realismo um $F=.68$ e um $p=.42$, e a presença global um $F=0.68$ e um $p=0.42$ (ver quadro 3).

Quadro 3

Resultados teste de efeitos entre sujeitos - variáveis do sentido de presença.

\begin{tabular}{cccc}
\hline Origem & Variável & $F$ & Sig \\
\hline \multirow{3}{*}{ Condição } & Presença Espacial & 2.52 & .13 \\
& Envolvimento & 1.58 & .22 \\
& Realismo & .68 & .42 \\
& Presença Global & .68 & .42 \\
\hline
\end{tabular}

\section{DISCUSSÃO}

Não se observaram diferenças estatisticamente significativas nas estimativas das distâncias no ambiente real e virtual, sendo que é possível constatar que os participantes estimaram de forma idêntica as distâncias em ambos os ambientes. Porém, ao contrário dos nossos resultados, estudos anteriores (Kelly, Siegel, \& Sjouland, 2014; Li, Philips, \& Durgin, 2011) concluíram que as distâncias egocêntricas são normalmente subestimadas por medidas verbais. No nosso estudo, como não houve diferenças estatisticamente significavas, podemos verificar que a assertividade sobre as estimativas das distâncias foi semelhante entre as condições.

De uma forma geral, a partir das análises estatísticas, foi possível evidenciar que a presença de membros virtuais, isto é, de mãos virtuais influenciou negativamente o envolvimento. Isto poderá ter acontecido devido aos participantes apenas verem umas mãos flutuantes, pois possivelmente se eles vissem um avatar completo os resultados poderiam ser diferentes. Por outro lado, os participantes na condição "sala virtual com membros virtuais" obtiveram médias superiores aos participantes na condição "sala virtual sem membros virtuais" no realismo e na presença espacial, ou seja, sentiram a experiência como mais realista e sentiram-se mais presentes no espaço quando visualizavam os membros virtuais. Contudo, na presença global os participantes na condição "sala virtual sem membros virtuais" obtiveram maior média, ou seja, quando considerado o realismo, o envolvimento e a presença espacial como um todo, os participantes que não visualizaram os membros virtuais expressaram maior sentimento de presença. Podemos assim inferir que a presença das mãos virtuais influencia negativamente o sentimento de presença no ambiente virtual, o que contraria a literatura que menciona que quem vê um self-avatar apresenta um maior sentido de presença e por conseguinte apresenta maior assertividade nas estimativas. A partir dos nossos resultados, verificámos que não houve diferenças significativas nas estimativas das distâncias entre a condição "sala virtual sem membros virtuais" e a condição "sala virtual com membros virtuais", podendo observar-se a partir da análise estatística que não houve efeitos estatísticos significativos relativamente às variáveis do sentido de presença tendo em conta as condições. Assim, apesar dos resultados do estudo de Mohler e Bülthoff (2008) fornecerem evidência de que se os participantes forem capazes de explorar um avatar totalmente articulado de si, cometerão menos erros nas suas avaliações subsequentes de distância egocêntrica, os nossos resultados 
não fornecem essa evidência.

Um aspeto interessante no campo do estudo de perceções das distâncias é que estudos recentes sugerem que o campo de visão pequeno nos sistemas HDM's não é explicação para a subestimação desde que os participantes sejam capazes de olhar ao redor do ambiente (Darken,et al., 1999; Creem-Regehr, $t$ al., 2008). Isto poderia se verificar no nosso estudo, pois os participantes apesar de não poder mover o corpo, tiveram liberdade para mover a cabeça, de maneira a observar da melhor forma a sala em que se encontravam.

\section{CONCLUSÕES}

Relativamente à nossa questão principal "será que existe maior subestimação das distâncias espaciais na condição "sala real" ou na condição "sala virtual", através dos resultados concluímos que não houve diferenças estatisticamente significativas nas estimativas das distâncias referentes a ambas as condições, ou seja, em ambas as condições os indivíduos estimaram as distâncias de forma idêntica. Assim, como não houve diferenças estatisticamente significativas nas estimativas das distâncias não existe um ambiente onde os participantes avaliam com maior assertividade as distâncias. E por conseguinte, o ambiente virtual pode ser usado para a perceção das distâncias.

Comparámos os dois grupos da condição "sala virtual", isto é, o grupo com membros virtuais e o grupo sem membros virtuais, de forma a observar se os participantes que visualizaram os membros virtuais na realidade virtual percecionaram as distâncias com maior assertividade. Através dos nossos resultados, concluímos que a presença dos membros virtuais influenciou negativamente o sentido de presença no ambiente virtual, sendo que os participantes descreveram um menor envolvimento e uma menor presença global na condição "sala virtual com membros virtuais”, contudo, verificámos que não foram encontradas diferenças significativas entre as estimativas das distâncias. Assim, concluímos que, no nosso estudo, não foi encontrada uma relação entre o sentido de presença e as estimativas verbais das distâncias.

Uma limitação que podemos apontar ao nosso estudo centra-se no facto de os participantes só visualizarem umas mãos flutuantes. Assim, recomenda-se que em futuros estudos se devem tomar medidas para acautelar esta influência através de um estudo mais cuidado acerca dos membros virtuais e do self-avatar. Outra limitação é que a nossa amostra é reduzida e, por isso, os nossos resultados não podem ser generalizados a toda a população. Estudos futuros deverão alargar a dimensão da amostra para chegar a resultados mais conclusivos.

Agradecimentos:

Nada declarado.

\section{Conflito de Interesses:}

Nada declarado.

\section{Financiamento:}

Nada declarado.

\section{REFERÊNCIAS}

Alexandrova, I. V., Teneva, P. T., Rosa, S., Kloos, U., Bülthoff, H. H. \& Mohler, B. J. (2010). Egocentric distance judgments in a large screen display immersive virtual environment. Association for Computing Machinery. 57-60. doi: 10.1145/1836248.1836258

Andre, J. \& Rogers, S. (2006). Using verbal and blind-walking distance estimates to investigate the two visual systems hypothesis. Perception \& Psychophysics, 68(3), 353-361. doi: 10.3758/BF03193682

Blascovich, J. J. \& Loomis, J. M. (1999). Immersive virtual environment technology as a basic research tool in psychology. Behavior Research Methods, Instrumets, \& 
Computers, 31(4), 557-564. doi: 10.3758/ BF03200735

Creem-Regehr, S. H., Gooch, A. A., Thompson, W. B. \& Willemsen, P. (2008). Effects of stereo viewing conditions on distance perception in virtual environments. Presence, 17(1), 91-101. doi: 10.1162/pres.17.1.91

Creem-Regehr, S. H., Mohler, B. J. \& Thompson, W. B. (2006). The influence of feedback on egocentric distance judgments in real and virtual environments. APGV, 9-14. doi: 10.1145/1140491.1140493

Creem-Regehr, S.H., Stefanucci, J.K., Thompson, W.B., Nash, N. \& McCardell, M. (2015). Egocentric Distance Perception in the oculus rift (DK2). ACM, 47-50. doi: $10.1145 / 2804408.2804422$

Darken, R. P., Krebs, W. K., McCarley, J. S., Rowland, J, H. \& Sinai, M. J. (1999). Egocentric distance perception in a virtual environment using a perceptual matching task. Proceedings of the Human Factors and Ergonomics Society Annual Meeting, 43(22). doi: 10.1177/154193129904302219

Jung, E., Takahashi, K., Watanabe, K., Rosa, S., Butz, M., Bülthoff, H.H. \& Meilinger, T. (2016). The influence of human body orientation on distance judgments. Frontiers in Psychology, 7. doi: 10.3389/fpsyg.2016.00217 Hammel, W. W., Kelly, J. W., Siegel, Z. D. \& Sjouland, L. A. (2014). Recalibration of perceived distance in virtual environments occurs rapidly and transfers asymmetrically across scale. IEEE Transactions on visualization and computer graphics, 20(4), 588-595. doi: 10.1109/TVCG.2014.36

Interrante, V., Ries, B., Lindquist, J. \& Anderson, L. (2008). Elucidating factors that can facilitate veridical spatial perception in immersive virtual environments. Presence, 17(2), 176-198. doi: 10.1162/pres.17.2.176

Knapp, J. M. \& Loomis, J. M. (2004). Limited field of view of head-mounted displays is not the cause of distance underestimation in virtual environments. Presence, 13(5),
572-577. doi: 10.1162/1054746042545238

Li, Z., Philips, J. \& Durgin, F.H. (2011). The underestimation of egocentric distance: evidence form frontal matching tasks. Atten Percept Psychophys, 73(7). 2205-2217. doi: 10.3758/s13414-011-0170-2

Matsushima, E.H., Vaz, A.M., Cazuza, R.A. \& Filho, N.P.R. (2014). Independence of egocentric and exocentric direction processing in visual space. Psychology $\mathcal{E}$ Neuroscience, 7(3). 277-284. doi: 10.3922/j. psns.2014.050

Mohler, B.J. \& Bülthoff, H.H. (2008). A full-body avatar improves egocentric distance judgments in an immersive virtual environment. ACM. doi: 10.1145/1394281.1394323

Renner, R.S., Velichkovsky, B.M. \& Helmert, J.R. (2013). The perception of egocentric distances in virtual environments - a review. ACM Computing Surveys, 46(2). doi: 10.1145/2543581.2543590

Riecke, B. E., Behbahani, P. A. \& Shaw, C. D. (2009). Display size does not affect egocentric distance perception of naturalistic stimuli. Proceedings of the 6th Symposium on Applied Perception in Graphics and Visualization, 15-18. doi: 10.1145/1620993.1620997

Steed, A., Pan, Y., Zisch, F. \& Steptoe, W. (2016). The Impact of a Self-Avatar on Cognitive Load in Immersive Virtual Reality. IEEE Virtual Reality (VR). doi: 10.1109/ VR.2016.7504689

Takahashi, K., Meilinger, T., Watanabe, K. \& Bülthoff, H. H. (2013). Psychological influences on distance estimation in a virtual reality environment. Frontiers in Human Neuroscience, 7. doi: 10.3389/ fnhum.2013.00580

Vasconcelos-Raposo, J., Bessa, M, Melo, M., Barbosa, L., Rodrigues, R., Teixeira, C. M., Cabral, L. \& Augusto Sousa, A. (2016). (IPQ) Adaptation and Validation of the Igroup Presence Questionnaire in a 
Portuguese sample. Presence: Teleoperators $\mathcal{E}$ Virtual Environments, 25(3).

Westhoven, M., Paul, D. \& Alexander, T. (2016). Head turn scaling below the threshold of perception in immersive virtual environments. VRST. 77-86. doi: $10.1145 / 2993369.2993385$
Ziemer, C. J., Plumert, J. M, Cremer, J. F. \& Kearney, J. K. (2009). Estimating distance in real and virtual environments: does order make a difference?. Atten Percept Psychophys, 71(5), 1095-1106. doi: 10.3758/ APP.71.5.109 